\title{
MicroRNA-197 promotes proliferation and inhibits apoptosis of gallbladder cancer cells by targeting insulin-like growth factor-binding protein 3
}

\author{
Li Tong $^{A, D}$, Jinglin Cheng ${ }^{A, C}$, Heping Zuo ${ }^{B}$, Jingrong Li ${ }^{C}$ \\ The Second Affiliated Hospital of Anhui Medical University, Hefei, China \\ A - research concept and design; B - collection and/or assembly of data; C - data analysis and interpretation; \\ $D$ - writing the article; $E$ - critical revision of the article; $F$ - final approval of the article
}

\section{Address for correspondence}

Jinglin Cheng

E-mail: ChengJingLin082828@163.com

\section{Funding sources}

None declared

Conflict of interest

None declared

\section{Acknowledgements}

We would like to acknowledge the reviewers

for their helpful comments on this paper.

Received on August 28, 2020

Reviewed on January 11, 2021

Accepted on March 24, 2021

Published online on June 11, 2021

Cite as

Tong L, Cheng J, Zuo H, Li J. MicroRNA-197 promotes proliferation and inhibits apoptosis of gallbladder cancer cells by targeting insulin-like growth factor-binding protein 3. Adv Clin Exp Med. 2021;30(7):661-672.

doi:10.17219/acem/134833

DOI

10.17219/acem/134833

\section{Copyright}

Copyright by Author(s)

This is an article distributed under the terms of the

Creative Commons Attribution 3.0 Unported (CC BY 3.0)

(https://creativecommons.org/licenses/by/3.0/)

\begin{abstract}
Background. Gallbladder cancer (GBC) is one of the common malignant tumors of the biliary tract. There is no report that miR-197 is involved in $\mathrm{GBC}$.

Objectives. The relationship between miR-197 expression and survival time of GBC patients was analyzed. Furthermore, the role and mechanism of miR-197 in GBC was explored.

Materials and methods. A total of $39 \mathrm{GBC}$ patients (21 males, 18 females; average age $56.1 \pm 8.5$ years) were included from December 2013 to November 2014. All patients were admitted to our hospital for surgical treatment (excluding patients with preoperative chemotherapy). The expression of miR-197 in GBC tissues was examined, and the relationship between miR-197 and patient survival time was analyzed. Cell Counting Kit-8 (CCK-8) and colony formation assays were used to detect cell proliferation. Flow cytometry and TUNEL staining were used to detect apoptosis. Expressions of proteins related with proliferation and apoptosis were detected. The target of miR-197 was predicted through bioinformatics website and verified using the dual luciferase reporter gene assay. The target gene was interfered to so that the effect of miR-197 on the regulation of $G B C$ cell proliferation and apoptosis could be observed.
\end{abstract}

Results. MiR-197 was highly expressed in GBC tissues, and the expression was closely related to the poor prognosis of GBC. Downregulation of miR-197 inhibited the proliferation and promoted the apoptosis of GBC cells; it also decreased the expressions of proliferation-related proteins p-ERK1/2 and p-AKT, and increased that of apoptosis pathway-related proteins $\mathrm{Bax} / \mathrm{BCl}-2$ and $\mathrm{c}-$ caspase-3. The upregulation of miR-197 induced an opposite trend. MiR-197 directly regulated /GFBP3.

Conclusions. Our study proved that the expression of miR-197 is closely related to the poor prognosis of GBC. The miR-197-IGFBP3 axis regulates the proliferation and apoptosis of GBC cells. Downregulation of miR-197 inhibited the proliferation and promoted the apoptosis of GBC cells, indicating potential therapeutic effects.

Key words: apoptosis, proliferation, gallbladder carcinoma, IGFBP3, miR-197 


\section{Background}

Gallbladder cancer (GBC) is the most common malignant tumor of the biliary tract, and ranked $6^{\text {th }}$ among all digestive system cancers by incidence. ${ }^{1}$ It is highly malignant and prone to both recur and metastasize, with a five-year survival rate between $5 \%$ and $15 \%$. $^{2,3}$ Therefore, it is crucial to clarify the pathogenesis of GBC. It is derived from the gallbladder mucosa, and studies have shown that the occurrence and development of GBC are closely related to the activation of the proliferation and inhibition of apoptosis pathways. ${ }^{4}$ At present, surgical resection remains an effective method to treat $\mathrm{GBC}$; however, due to the poor prognosis of $\mathrm{GBC}$ most patients do not have the opportunity to undergo radical resection. ${ }^{5}$ Therefore, further research focusing on the molecular mechanism is urgently needed to develop more effective therapeutic approaches for GBC treatment.

Recent studies have shown that microRNAs (miRs) are integrally involved in the onset and development of tumors. The miRs function as critical regulators of cancer occurrence, proliferation and apoptosis and certain miRs; namely, miR-20a, miR-182 and miR-155 have been described as potential GBC biomarkers. ${ }^{6}$ Furthermore, recent literature has revealed that miR-197 has various patterns of expression and effects in different tumors. For example, miR-197 is downregulated in osteosarcoma, colorectal cancer and breast cancer, while being upregulated in lung, ovarian and pancreatic cancer, and hepatocellular carcinoma. ${ }^{7}$ Through bioinformatics analysis, we found that miR-197 was significantly upregulated in GBC tissues, ${ }^{8}$ but the mechanism remains unclear.

\section{Objectives}

This study intends to explore the underlying mechanism of miR-197 in regulating GBC.

\section{Materials and methods}

\section{Clinical samples}

A total of 39 GBC patients from The Second Affiliated Hospital of Anhui Medical University, Hefei, China, were included from December 2013 to November 2014, with an average age of $56.1 \pm 8.5$ years (21 males and 18 females). All patients were admitted to our hospital for surgical treatment (patients with preoperative chemotherapy were excluded). The overall survival (OS) time of patients was followed for up to 5 years. Tumor samples and adjacent normal tissue biopsies were taken during the operation. This study was approved by the institutional Ethics Committee and all patients gave informed consent prior to undergoing any procedure.
This study was approved by Ethics Committee of The Second Affiliated Hospital of Anhui Medical University. All patients provided written informed consent.

\section{Differential expression analysis of microarray data in GBC}

The GBC expression microarray, GSE104165, which contains $40 \mathrm{GBC}$ and 8 normal gallbladder tissue samples, was extracted from the Gene Expression Omnibus (GEO) database (https://www.ncbi.nlm.nih.gov/geo/). ${ }^{8}$ Differential expression analyses were performed using the $\mathrm{R}$ language "limma" package (https://www.r-project.org), with log fold change $(\mathrm{FC})>2$ and $\mathrm{p}$-value $<0.05$ regarded as the threshold of differentially expressed genes.

\section{Cell culture}

Human gallbladder epithelial cells (HGBEC) and 5 gallbladder cancer cell lines (GBC-SD, EH-GB1, NOZ, SGC-996, and MZ-CHA-1; purchased from the Cell Bank of Shanghai Institute of Biochemistry and Cell Biology, Chinese Academy of Sciences, Shanghai, China) were cultured in Dulbecco's modified Eagle's medium (DMEM; Gibco, Carlsbad, USA) with $10 \%$ fetal bovine serum (FBS), $1 \%$ penicillin (100 $\mathrm{U} / \mathrm{L})$ and streptomycin $(100 \mathrm{mg} / \mathrm{L})$ in a $5 \% \mathrm{CO}_{2}$ incubator at $37^{\circ} \mathrm{C}$. Sub-culture was performed every $2-3$ days.

\section{Cell transfection}

The synthetic miR-197 mimic (miR-197) and miR-197 inhibitor (in-miR-197), together with corresponding mimic and inhibitor controls, were purchased from GenePharma Inc. (Shanghai, China). The cells were seeded in six-well plates and transfected with miR-197, in-miR-197 or their negative controls using Lipofectamine ${ }^{\circledR} 2000$ transfection reagent (Invitrogen, Thermo Fisher Scientific, Inc., Waltham, USA), based on the manufacturer's guidelines. Briefly, $100 \mathrm{pmol}$ of mimic or inhibitor was added to the mixture of $250 \mu \mathrm{L}$ of Opti-MEM (Gibco, Thermo Fisher Scientific, Inc.) and $5 \mu \mathrm{L}$ of Lipofectamine ${ }^{\circledR} 2000$. The mixture was then added into cells ensuring the final concentration of miR-197, inmiR-197 or corresponding controls is $50 \mathrm{nM}$, and the culture medium was changed $6 \mathrm{~h}$ after transfection. After a 48-hour period, the cells were collected for subsequent experiments.

Insulin-like growth factor binding protein 3 (IGFBP3) siRNA (siIGFBP3) and its corresponding negative control were constructed using GENECHEM and siIGFBP3 transfection was performed. In brief, the medium was removed from the six-well plate, and $2 \mathrm{~mL}$ of serum-containing medium was added without antibiotics into each well. Then, $100 \mu \mathrm{L}$ of Opti-MEM ${ }^{\circledR}$ (Gibco, Thermo Fisher Scientific, Inc.) $+2000 \mathrm{ng}$ of siIGFBP3 plasmid $+10 \mu \mathrm{L}$ of transfection reagent was added (Invitrogen, Thermo Fisher Scientific, Inc.) to each well of the plate, the plate was vortexed and incubated at room temperature for $10 \mathrm{~min}$. Next, 
the transfection mixture was added to a six-well plate and continued to incubate in a $5 \% \mathrm{CO}_{2}$ incubator. The mixture was added to the cells, and the medium changed after $6 \mathrm{~h}$. After $48 \mathrm{~h}$, the cells were collected for further experiments.

\section{Dual luciferase reporter gene assay}

We applied the biological database (http://www.targetscan.org/vert_71/) to confirm the relationship between miR-197 and its putative binding sequence of the IGFBP3 gene. The outcome indicates that the IGFBP3 gene is one of the target genes of miR-197. Fragments from IGFBP3 and flanking sequences on each side were synthesized with a short extension containing cleavage sites for XhoI (5'-end) and NotI (3'-end), while the second fragment, containing mutated binding site sequences, was also synthesized. The 2 constructs were termed as WT (wild-type gene) and MT (mutant genes). The fragments were cloned into the psiCHECK $^{\mathrm{TM}}-2$ vector (Promega, Madison, USA). Then, 293 T-cells (purchased from Cell Bank of the Shanghai Institute of Biochemistry \& Cell Biology) were seeded in a six-well plate (Corning Inc., Corning, USA) at a density of $1 \times 10^{5}$ cells per well, and the wild-type IGFBP3 UTR or mutant IGFBP3 UTR plasmids (Hanyin, Shanghai, China) with miR-197 mimic using Invitrogen ${ }^{\text {TM }}$ Lipofectamine 2000 (Invitrogen) were transfected according to the manufacturer's instructions. After $24 \mathrm{~h}$ of incubation, the luciferase activity of the WT and mutated IGFBP3 UTR was detected using the dual-luciferase reporter assay system (Promega).

\section{Quantitative real-time PCR}

Total RNA was extracted from GBC tissues and cells using an RNA extraction kit (Invitrogen); PrimeScript reverse transcription (RT) kit was applied to reverse-transcribe RNA into cDNA. Fluorescent quantitative polymerase chain reaction (qPCR) was performed with $S Y B R^{\circledR}$ Premix Ex TaqTM II solution kit using the ABI PRISM ${ }^{\circledR} 7300$ system (Applied Biosystems, Foster City, USA). The primers for real-time PCR (RT-PCR) are shown in Table 1. U6 was used as the internal reference for miR-197, and GAPDH was applied as the endogenous control for mRNA. The data was analyzed using the $2^{-\Delta \Delta \mathrm{Ct}}$ method. ${ }^{9}$

Table 1. Primers (mRNA) for real-time PCR

\begin{tabular}{|c|c|}
\hline Name & Sequences \\
\hline \multirow{2}{*}{ miR-197 } & 5'-TTCACCACCTTCTCCACCCAGC-3' (forward) \\
\hline & 5'-TGCACTGGTCCAAGCTCTAACC-3' (reverse) \\
\hline \multirow{2}{*}{ U6 } & 5'-CTCGCTTCGGCAGCACA-3" (forward) \\
\hline & 5'-AACGCTTCACGAATTTG CGT-3' (reverse) \\
\hline \multirow{2}{*}{ IGFBP3 } & 5'-GAGGGCGACACTGCTTTTTC-3' (forward) \\
\hline & 5'-CCAGCT CCAGGAAATGCTAG-3' (reverse) \\
\hline \multirow{2}{*}{ GAPDH } & 5'-GAAGGTGAAGGTCGGAGTC-3' (forward) \\
\hline & 5'-AAGATGGTGATGGGATTTC-3' (reverse) \\
\hline
\end{tabular}

\section{Western blotting analysis}

SGC-996 and MZ-CHA-1 cells were washed twice with ice-cold PBS, then incubated in lysis buffer $(150 \mathrm{mM} \mathrm{NaCl}, 1 \%$ Nonidet P-40, 0.1\% SDS, $2 \mathrm{mg} / \mathrm{mL}$ of aprotinin, and $1 \mathrm{mM}$ phenylmethylsulfonyl fluoride (PMSF)) for $30 \mathrm{~min}$. The supernatants were centrifuged at $12,000 \times \mathrm{g}$ for $15 \mathrm{~min}$ at $4^{\circ} \mathrm{C}$, and retained for protein extraction. The protein $(30 \mu \mathrm{g})$ was subjected to sodium dodecyl sulfate salt - polyacrylamide gel electrophoresis (SDS-PAGE 10\% (w/v) acrylamide gel) and blotted onto polyvinylidene difluoride (PVDF) membranes, which were washed for $1 \mathrm{~h}$ with a blocking solution. The membranes were incubated overnight at $4^{\circ} \mathrm{C}$ with anti-IFGBP3 (1:1000; ab220429; Abcam Inc., Cambridge, USA), anti-ERK1/2 (1:1000; 9102; Cell Signaling Technology (CST), Beverly, USA), anti-p-ERK1/2 (1:1000; 4370; CST), anti-AKT (1:1000; 4691; CST), anti-p-AKT $(1: 1000 ; 4060 ;$ CST), and anti-glyceraldehyde-3-phosphate dehydrogenase (GAPDH) (1:2000; ATA29666; Zhongshan Golden Bridge Biotechnology, Beijing, China) antibodies. The next day, membranes were incubated with a secondary anti-rabbit or antimouse horseradish peroxidase (HRP)-conjugated antibody (1:1000; Zhongshan Golden Bridge Biotechnology). Finally, the relative expression levels of protein bands were analyzed using ImageJ software (National Institutes of Health, Bethesda, USA).

\section{TdT-mediated dUTP nick-end labeling (TUNEL) staining}

Based on the instructions of the Biyuntian TUNEL Apoptosis Detection Kit, genomic DNA was cut, and on the exposed 3'-OH end, a dUTP-GFP base was fixed that could be directly viewed under a microscope. The specific steps were as follows: cells were fixed with $4 \%$ paraformaldehyde and then permeabilized with $0.1 \%$ Triton X-100 for $2 \mathrm{~min}$. The TUNEL reaction mixture was added to react for $60 \mathrm{~min}$, and then with Converter-POD for $30 \mathrm{~min}$. After washing, the cells were stained in $100 \mu \mathrm{L}$ of DAPI nuclear staining reagent (Sigma-Aldrich, St. Louis, USA) for $10 \mathrm{~min}$. The stained cells were observed under the Olympus BX-53 fluorescence microscope and the images were captured using a digital camera (Olympus Corp., Tokyo, Japan) with a $\times 10$ magnification. Nine fields per slide were then randomly selected. The number of TUNEL-positive cells was counted and averaged on each field of view. The results were expressed as mean \pm standard error of the mean (SEM).

\section{Colony formation assay}

The colony formation assay is conducted in a six-well plate in which cells were seeded at a density of $8 \times 10^{2}$ cells/ well and cultured for 2 weeks, and then the colonies were 
fixed with $4 \%$ paraformaldehyde and stained with $0.1 \%$ crystal violet (Sigma-Aldrich) The number of colonies was counted, and the experiment was independently repeated 3 times to ensure accuracy.

\section{Flow cytometry}

The cells of each group were suspended, collected and washed twice with saline. After that, the cells were fixed with $70 \%$ ethanol. Then, $100 \mu \mathrm{L}$ of cell suspension was added to a 5 -mL culture tube, and subsequently $5 \mu \mathrm{L}$ of FITC-labeled Annexin-V (1:20, C1062S; Biotechnology, Shanghai, China) and $5 \mu \mathrm{L}$ of propidium iodide (PI) (1:20, $\mathrm{C} 1062 \mathrm{~S})$ were added, mixed and incubated in the dark at room temperature for $15 \mathrm{~min}$. After incubation, $400 \mu \mathrm{L}$ of $1 \mathrm{X}$ binding buffer was added, ensuring precise and accurate movements the whole procedure. Next, cell apoptosis was detected using flow cytometry (BD FacsCanto II; Becton Dickinson, Franklin Lakes, USA).

\section{Cell Counting Kit-8 assay}

The transfected cells were seeded into 96-well plates $\left(1 \times 10^{3}\right.$ cells/well) with added Cell Counting Kit-8 (CCK-8; Invitrogen) according to the manufacturer's protocol. Cell proliferation was detected through the optical density (OD) value $(570 \mathrm{~nm})$.

\section{Statistical analyses}

The IBM SPSS v. 21.0 software (IBM Corp. Armonk, USA) was utilized for data processing. The KolmogorovSmirnov test was used to check whether data was distributed normally. Data are presented as mean \pm standard deviation (SD). Comparisons between 2 groups were analyzed using a Student's t-test and comparisons among multiple groups were analyzed using one-way or two-way analysis of variance (ANOVA), followed by the Tukey's multiple comparisons test. Kaplan-Meier method was used for survival analysis. The p-value was calculated using a two-tailed test and $\mathrm{p}<0.05$ indicated a statistically significant difference.

\section{Results}

\section{MiR-197 was highly expressed in GBC tissues}

To date, there is no study that focuses on whether miR-197 regulates the occurrence and development of GBC. Through a bioinformatics analysis (http://www.ncbi.nlm. nih. gov/geo/), we found that miR-197 was highly expressed in GBC tissues (Fig. 1A). Furthermore, there is a significant upregulation of miR-197 in tumor tissues $(\mathrm{p}<0.05)$ when compared to healthy gallbladder tissue (Fig. 1B). Then, according to the median expression of miR-197, the 39 patients examined in this study were categorized into a high ( $\geq$ median miR-197 expression; $\mathrm{n}=19$ ) or low expression group (<median miR-197 expression; $\mathrm{n}=20$ ), and the baseline characteristics of different groups were compared (Table 2). The results showed that the expression of miR-197 was significantly correlated with T classification, also known as the depth of tumor invasion $(\mathrm{p}<0.05)$. Kaplan-Meier analysis showed that the expression of miR197 was closely related to the poor prognosis of the GBC patients (Fig. 1C). Additionally, compared with normal gallbladder cells (HGBEC), GBC cell lines (EH-GB1, NOZ, SGC-996, and MZ-CHA-1) had higher expression of miR197 ( $\mathrm{p}<0.05$ ), and of these cell lines, the upregulated expression of miR-197 in SGC-996 and MZ-CHA-1 cells was statistically significant (Fig. 1D).

Table 2. Correlation between clinicopathological characteristics and miR-197 expression in 39 patients with GBC

\begin{tabular}{|c|c|c|c|c|}
\hline \multirow{2}{*}{\multicolumn{2}{|c|}{ Characteristics }} & \multicolumn{2}{|c|}{ miR-197 expression (median expression 8.374) } & \multirow{2}{*}{ p-value } \\
\hline & & low & high & \\
\hline \multirow{2}{*}{ Age } & $<56$ & $11(28.2 \%)$ & $9(23.1 \%)$ & \multirow{2}{*}{0.634} \\
\hline & $>56$ & $9(23.1 \%)$ & $10(25.6 \%)$ & \\
\hline \multirow{2}{*}{ Gender } & male & $12(30.8 \%)$ & $9(23.1 \%)$ & \multirow{2}{*}{0.429} \\
\hline & female & $8(20.5 \%)$ & $10(25.6 \%)$ & \\
\hline \multirow{2}{*}{ T classification } & $1-2$ & $14(35.9 \%)$ & $6(15.4 \%)$ & \multirow{2}{*}{0.016} \\
\hline & $3-4$ & $6(15.4 \%)$ & $13(33.3 \%)$ & \\
\hline \multirow{2}{*}{ N classification } & 0 & $11(28.2 \%)$ & $11(28.2 \%)$ & \multirow{2}{*}{0.855} \\
\hline & $1-2$ & $9(23.1 \%)$ & $8(20.5 \%)$ & \\
\hline \multirow{2}{*}{ Distant metastasis } & no & $10(25.6 \%)$ & $9(23.1 \%)$ & \multirow{2}{*}{0.870} \\
\hline & yes & $10(25.6 \%)$ & $10(25.6 \%)$ & \\
\hline \multirow{2}{*}{ Pathologic stage } & $1-2$ & $9(23.1 \%)$ & $10(25.6 \%)$ & \multirow{2}{*}{0.634} \\
\hline & $3-4$ & $11(28.2 \%)$ & $9(23.1 \%)$ & \\
\hline
\end{tabular}



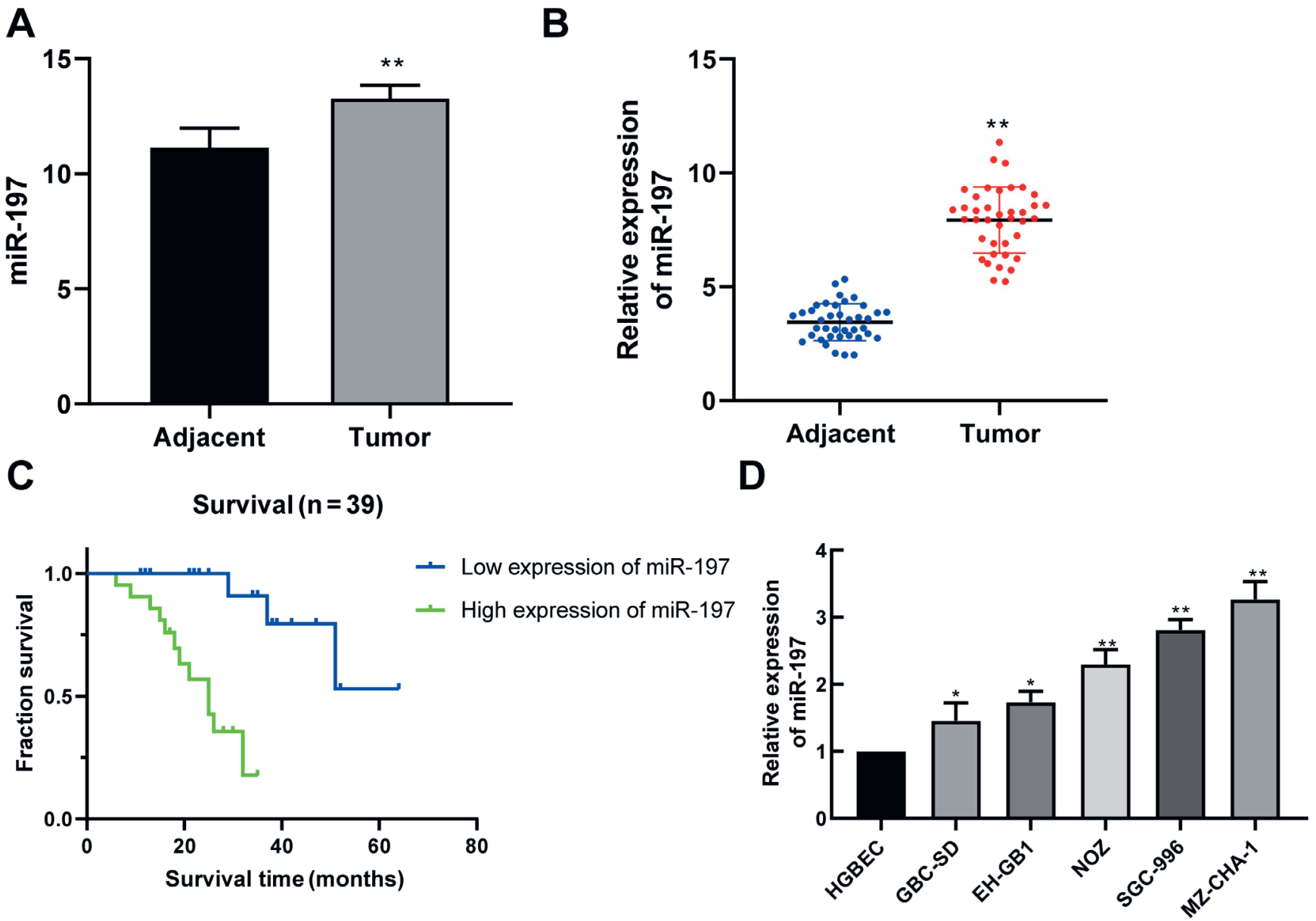

Fig. 1. MiR-197 is highly expressed in GBC tissues and cells. A. Bioinformatics analysis (http://www.ncbi.nlm.nih.gov/geo/) of the expression of miR-197 in GBC. ${ }^{* *}$ Compared with the adjacent group, $p<0.01$; B. The expression of miR-197 in GBC tissues and adjacent normal tissues was detected using qRT-PCR. U6 was used as endogenous control. ** compared with the adjacent group, $p<0.01$; C. Kaplan-Meier analysis observed the correlation between expression of miR-197 and survival; D. The expression of miR-197 in HGBEC, GBC-SD, EH-GB1, NOZ, SGC-996, and MZ-CHA-1 cell lines was detected using qRT-PCR, and U6 was used as endogenous control. ${ }^{*}$ compared with HGBEC group, $p<0.05$. Data represent the $m e a n \pm S D(n=3)$

\section{Downregulation of miR-197 impeded cell proliferation and promoted apoptosis of GBC cells}

To study the role of miR-197 in GBC cells, the miR-197 inhibitor in-miR-197 was applied to GBC cells, while a random sequence was used as a negative control (in-NC), and transiently transfected into SGC-996 and MZ-CHA-1 cell lines. After 48 h, miR-197 expression was significantly reduced in SGC-996 and MZ-CHA-1 cells (both $\mathrm{p}<0.01$; Fig. 2A). Then, CCK-8 and plate cloning experiments were used to test the effect of miR-197 on cell proliferation. These results demonstrated that in-miR-197 significantly inhibited cell proliferation compared with cells in the inNC group (Fig. 2B), with a concomitant significant reduction in the number of clones (both $\mathrm{p}<0.01$; Fig. 2C). We then observed reduced miR-197 in SGC-996 and MZ-CHA-1 cells resulted in a significant increase in both the apoptosis rate (both $\mathrm{p}<0.01$; Fig. 2D), and the number of TUNEL-positive cells (both $\mathrm{p}<0.01$; Fig. 2E). The above results showed that downregulation of miR-197 inhibited GBC cell proliferation and promoted apoptosis.

\section{MiR-197 regulated the expressions of proliferation pathway and apoptosis pathway-related proteins}

As mentioned above, downregulation of miR-197 inhibited the proliferation of GBC cells and promoted apoptosis. This prompted us to explore the regulatory role of miR-197 in proliferation/apoptosis signaling pathways. It was demonstrated that miR-197 was significantly elevated in MZ-CHA-1 cells after transfection with miR-197 (Supplementary Fig. 6A), causing a subsequent upregulation of p-ERK1/2, p-AKT and Bcl-2, and downregulated the expression of c-caspase-3 and Bax (Fig. 3A-C). Inhibition of miR-197 with in-miR-197 significantly downregulated the expression of $\mathrm{p}-\mathrm{ERK} 1 / 2, \mathrm{p}-\mathrm{AKT}$ and $\mathrm{Bcl}-2$, and upregulated the expression of c-caspase- 3 and Bax (Fig. 3A,B,D). From the data above, it is reasonable to conclude that upregulation of miR-197 stimulated ERK and AKT pathways, and inhibited the Bax/Bcl-2 and caspase-3 pathways, while downregulation of miR-197 showed the opposite effect. 
A

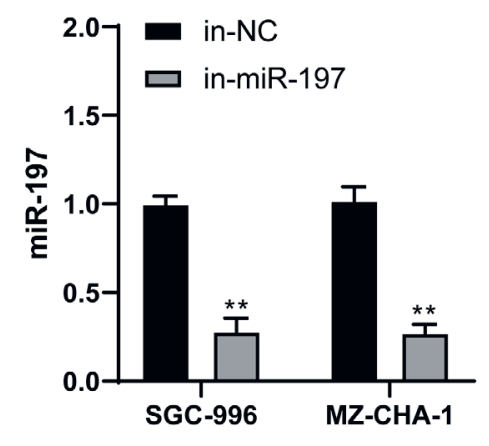

C

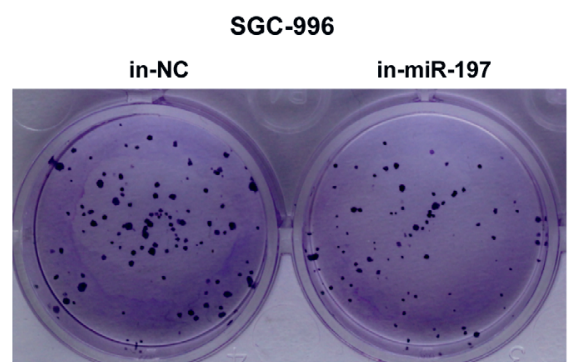

D

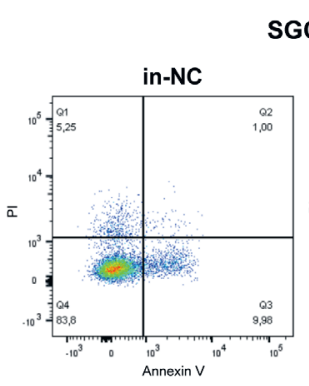

SGC-996
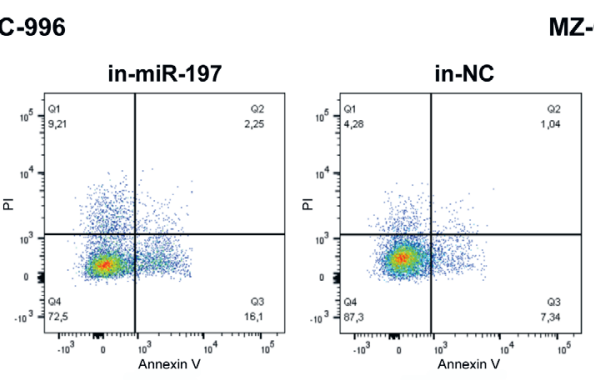

MZ-CHA-1
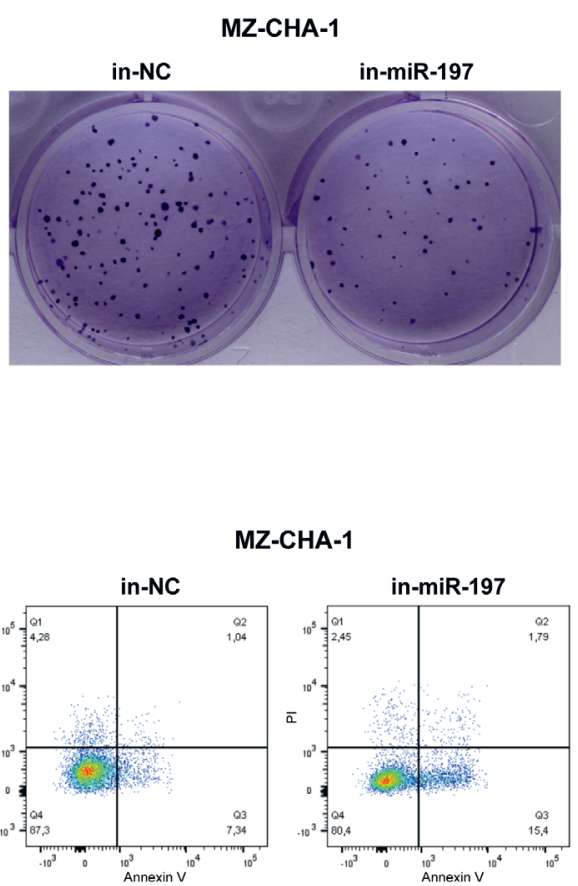

E

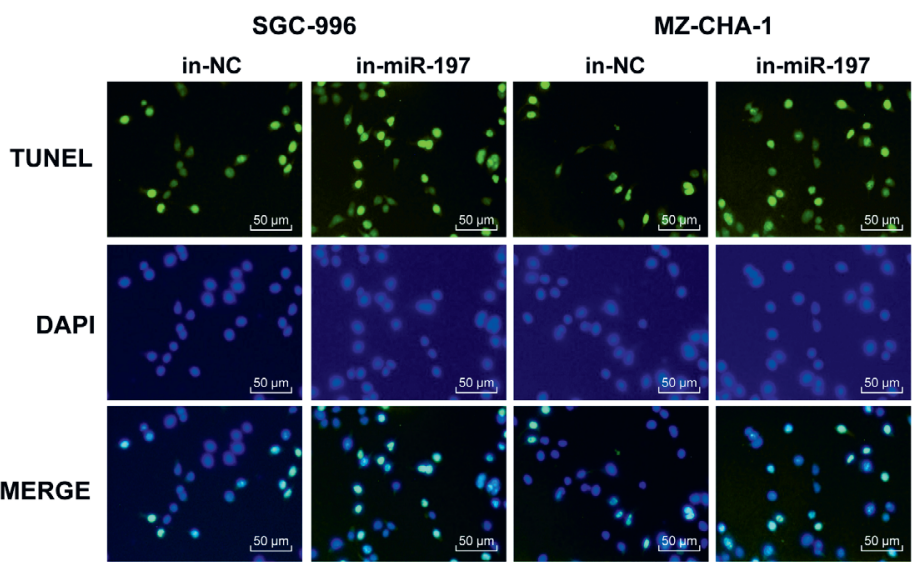

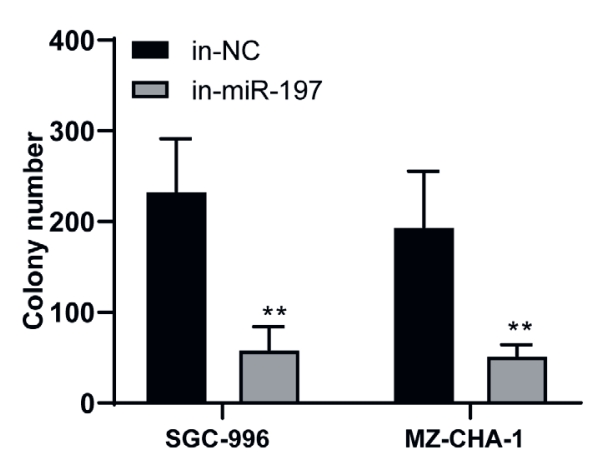

MZ-CHA-1
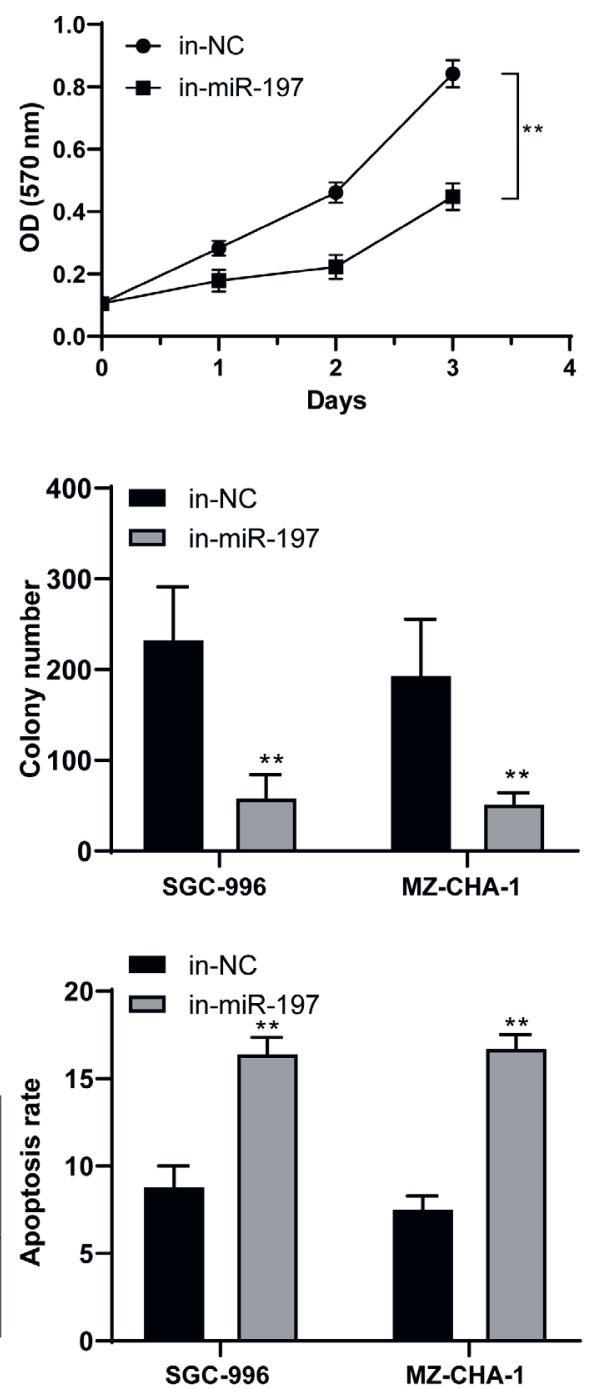

B

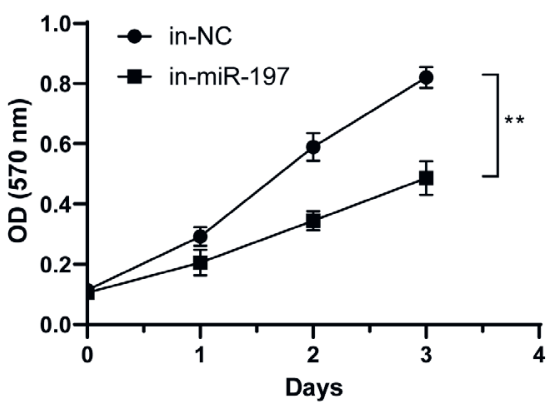



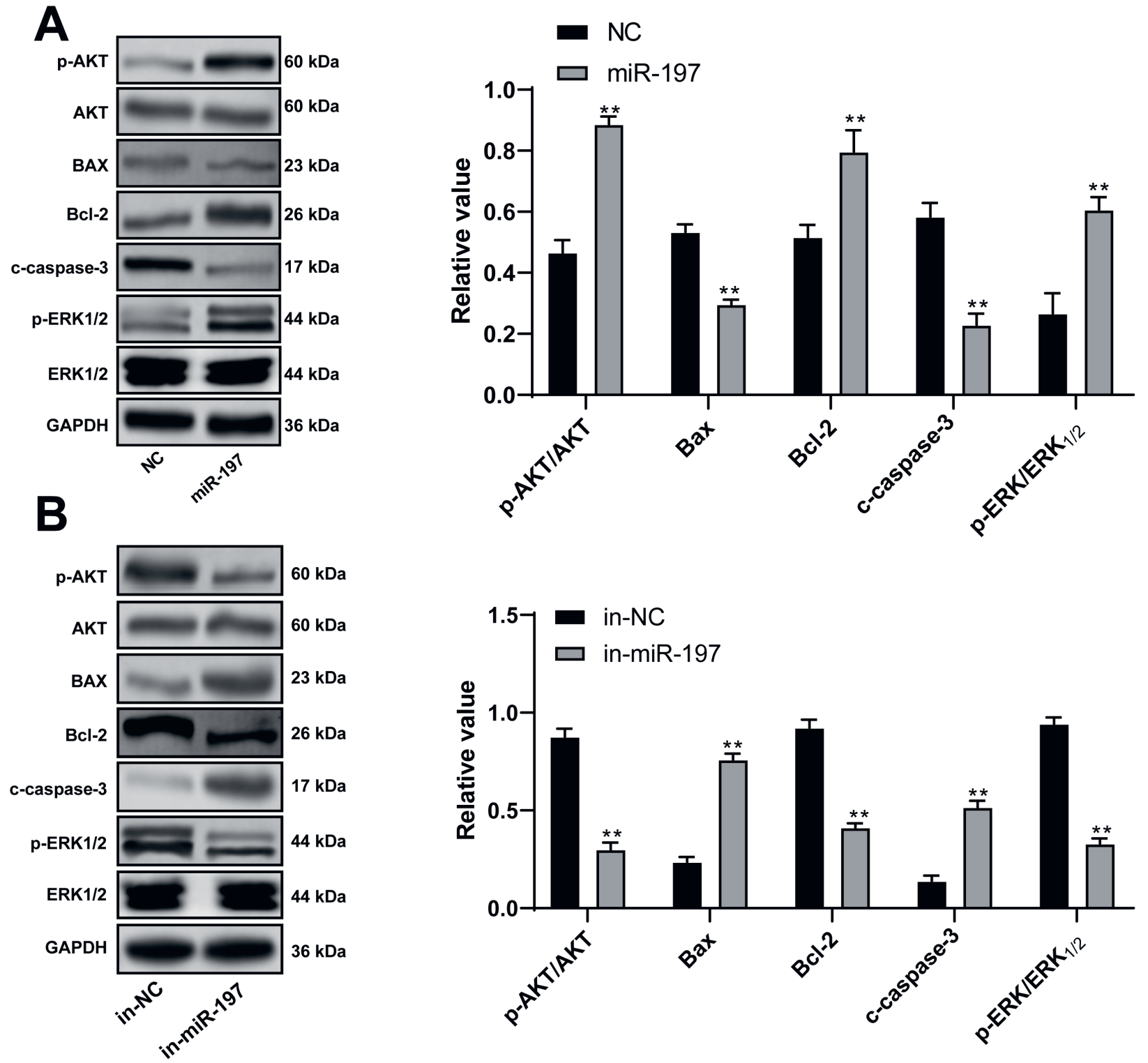

Fig. 3. MiR-197 regulates the expression of proliferation pathway proteins p-ERK1/2 and p-AKT and apoptosis pathway proteins Bax/BCl-2 and c-caspase-3. A and B. MZ-CHA-1 cells were transfected with miR-197/in-miR-197, and western blotting analysis was used to detect the expression of p-ERK1/2 (42KD), p-AKT (60KD), Bax (21KD), BCl-2 (26KD), and c-caspase-3 (17KD); C and D. Statistical analysis. Data represent the mean $\pm S D(n=3)$

${ }^{*}$ compared with NC/in-NC, $p<0.05$.

\section{MiR-197 inhibited the expression of IGFBP3}

MiRNA regulates cell proliferation and apoptosis by regulating its downstream target genes. Therefore, we predicted which mRNA may be targeted by miR-197 using an online database (http://www.targetscan.org/vert_71/) to further explore the mechanism of miR-197 regulation of GBC cell proliferation and apoptosis. The predicted results revealed that the IGFBP3 gene, which is closely related to tumor proliferation, is one of the target genes of miR197. The binding sites of IGFBP3 mRNA and miR-197 are shown in Fig. 4A. We used a dual luciferase reporter gene assay to verify this relationship. When co-transfected with the IGFBP3 WT reporter plasmid, miR-197 significantly reduced luciferase activity compared with the NC group, and the mutated reporter plasmid eliminated miR-197-mediated inhibitory effect on the luciferase activity (both $\mathrm{p}<0.05$ ) (Fig. 4B). These results demonstrated that miR197 and IGFBP3 mRNA closely interact.

In order to show whether miR-197 can affect the expression of IGFBP3 in GBC, the SGC-996 cell line was transfected with miR-197, in-miR-197 and their NCs; then, the expression of IGFBP3 at the mRNA and protein levels was examined. After transfection with miR-197, the mRNA level of IGFBP3 in SGC-996 cells was significantly reduced, while transfection with in-miR-197 caused a significant increase in the mRNA level of IGFBP3 (all p < 0.05; Fig. 4C). Upregulation of miR-197 caused a decrease in IGFBP3 protein expression, while downregulation of miR-197 exerted the reverse effect (all $\mathrm{p}<0.05$; Fig. 4D). Compared with 
A

IGFBP3 3'UTR 5' ..UUUAUAUUUCUGUUUGUGGUGAA...

hsa-miR-197

B

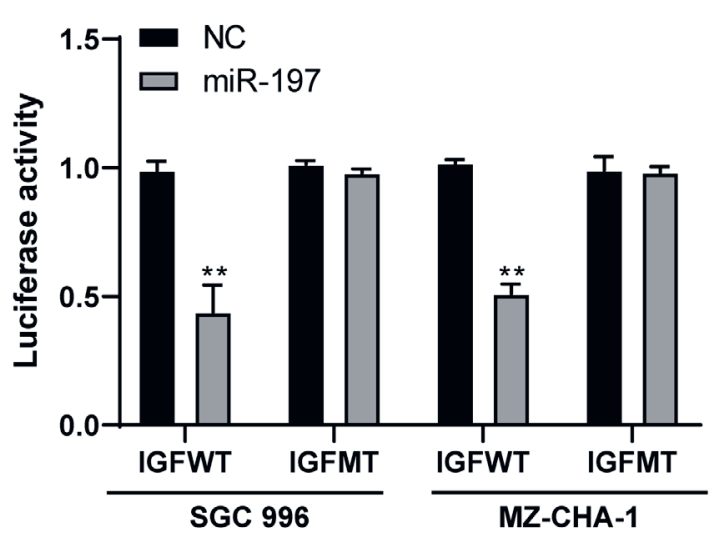

C

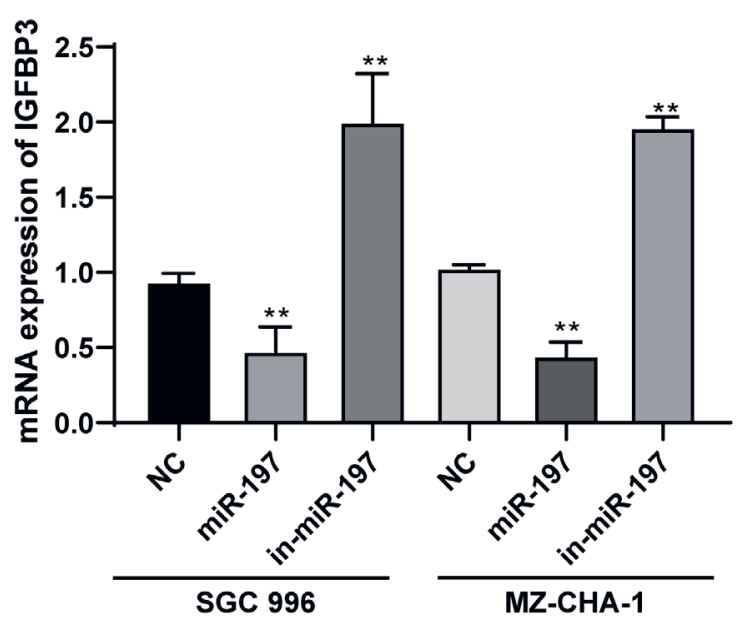

|||||| $\mid$

3' CGACCCACCUCUUCCACCACUU
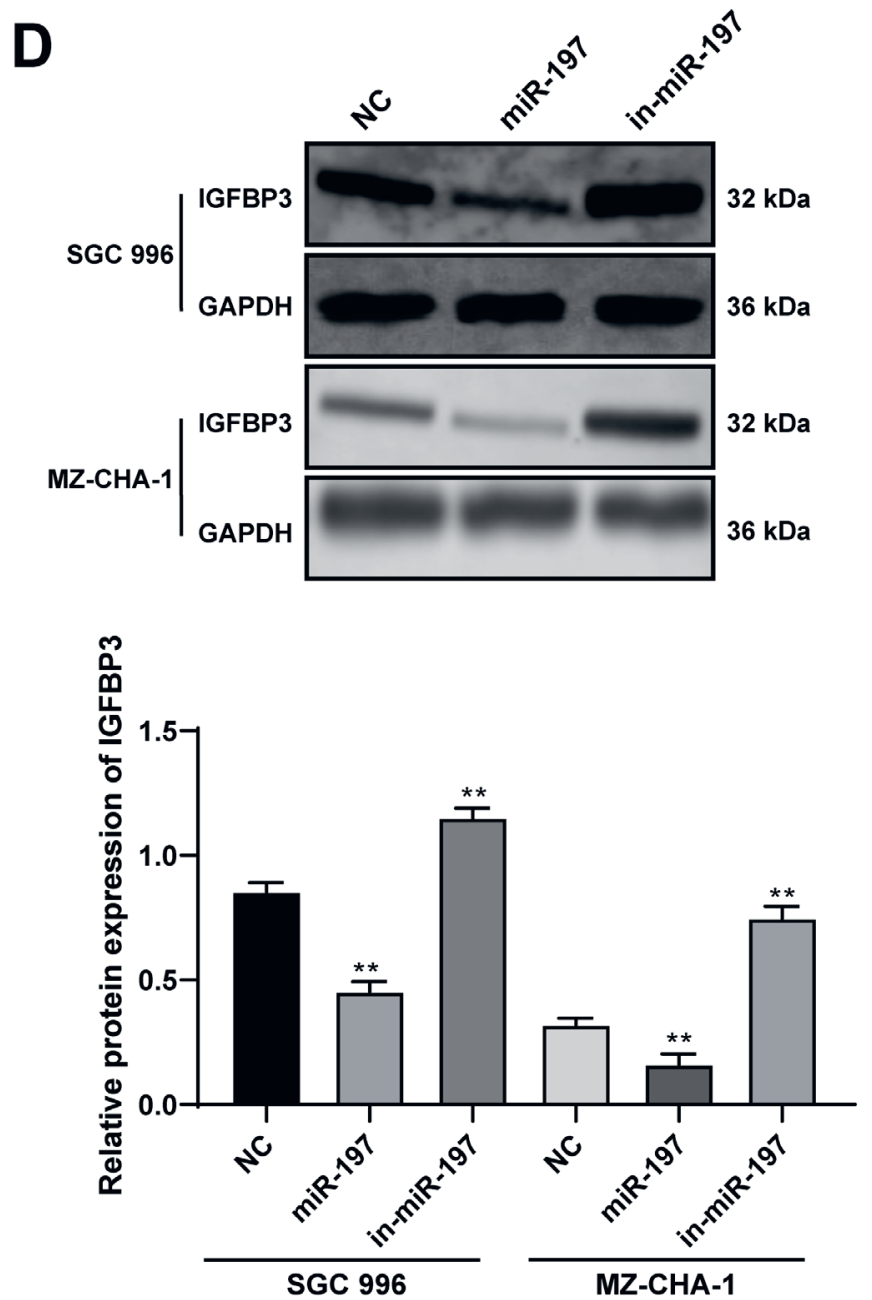

Fig. 4. MiR-197 inhibits the expression of IGFBP3. A. The miR-197 target sequence predicted in the 3'UTR of /GFBP3; B. SGC-996 and MZ-CHA-1 cells were CO-transfected with miR-197 and IGFBP3 WT or MT, and the luciferase activity was determined; C and D. SGC-996 and MZ-CHA-1 cells were transfected with miR-197/in-miR-197, and the expression of IGFBP3 (32KD) was detected using qRT-PCR and western blotting analysis. Data represent the mean \pm SD $(n=3)$

${ }^{*}$ compared with NC, $\mathrm{p}<0.05 ;{ }^{* *}$ compared with NC, $\mathrm{p}<0.01$.

the adjacent tissues, the IGFBP3 expression in tumor tissues was significantly reduced (Supplementary Fig. 6B). These results indicated that IGFBP3 was one of the target genes of miR-197 in GBC cells.

\section{The silGFBP3 could partially reverse the effect caused by miR-197 downregulation}

The above results indicated that IGFBP3 may participate in the miR-197-regulated proliferation and apoptosis of GBC cells, which prompted us to further explore its role. Firstly, we were able to effectively silence siIGFBP3 in MZCHA-1 cells (Fig. 5A,B). Then, transfection of the cells with miR-197 inhibitor combined with siIGFBP3 (in-miR197+siIGFBP3) increased the number of cell clones (Fig. 5C) when analyzed by the OD value (570 nm) (Fig. 5D). This group (in-miR-197+siIGFBP3) also had a lower apoptosis rate compared with the in-miR-197 group (Fig. 5E), suggesting that siIGFBP3 can partially inhibit the elevated apoptosis induced by miR-197 downregulation.

\section{Discussion}

Investigation on the pathophysiology of GBC is critical due to its high recurrence rate and poor prognosis. To date, there is no report on the role of miR-197 during the disease 

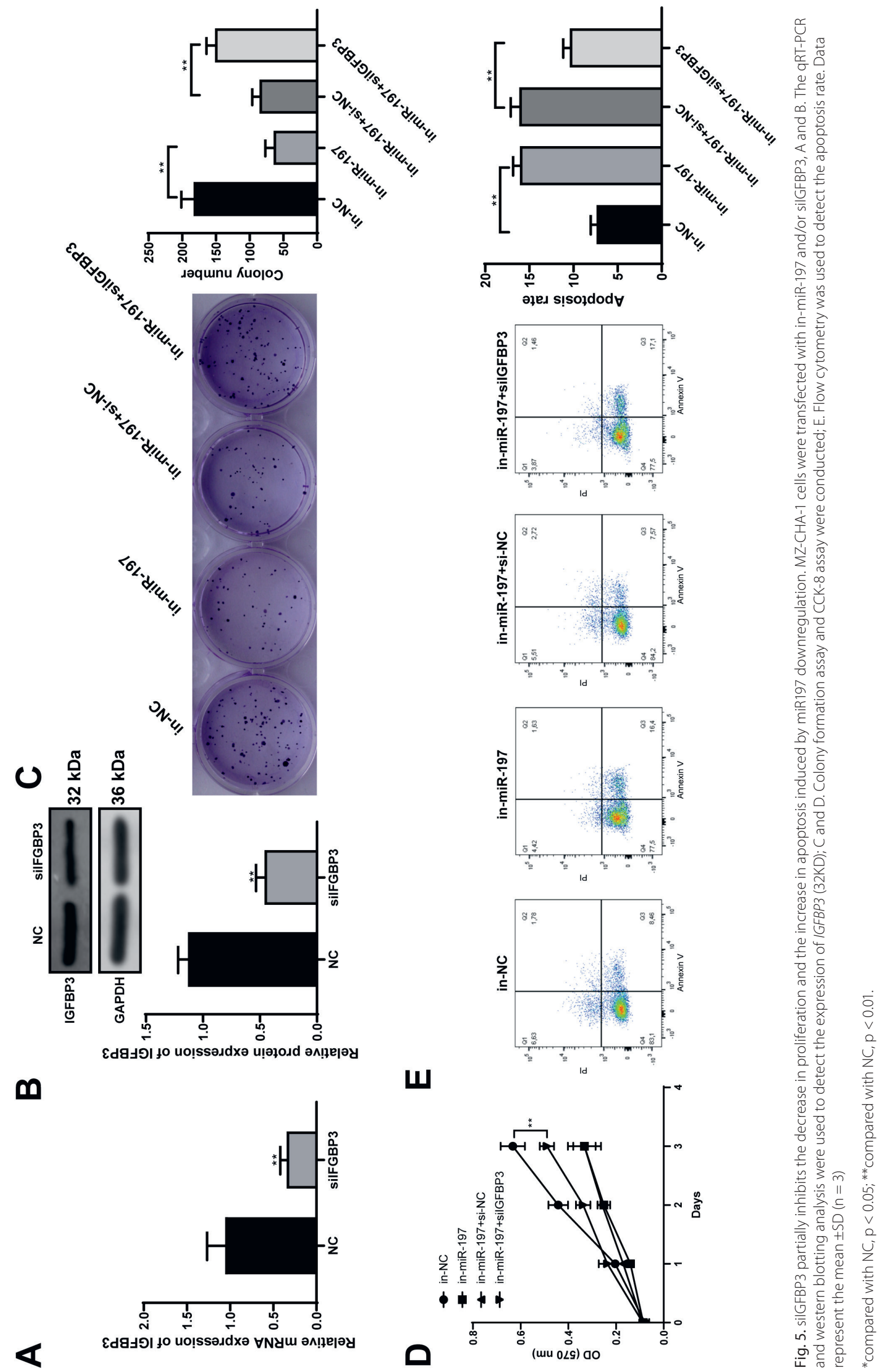

Ш
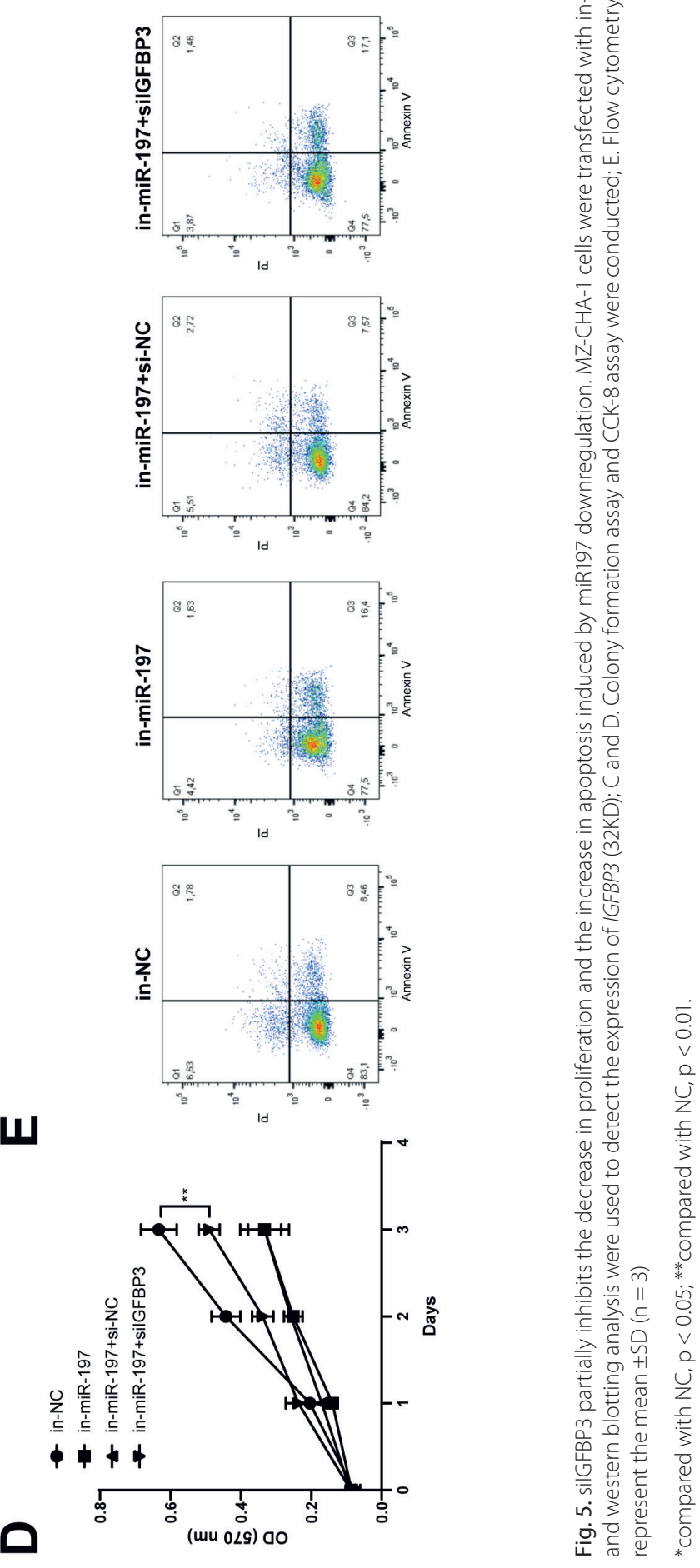
process. In this study, we found that miR-197 was highly expressed in GBC tissues, and the expression of miR-197 was closely related to the poor prognosis of GBC. MiR197 was highly expressed in GBC cells, and downregulation of miR-197 inhibited GBC cell proliferation and promoted apoptosis. It acts through decreasing the expression of proliferation-related proteins $\mathrm{p}$-ERK $1 / 2$ and $\mathrm{p}$-AKT, while increasing expression of apoptosis pathway-related proteins Bax/Bcl-2 and c-caspase-3. Upregulation of miR197, on the other hand, induced an opposite trend. MiR-197 directly regulated IGFBP3, as shown by silencing the gene using siIGFBP3, which partially inhibited the decrease of proliferation and the increase of apoptosis induced by downregulation of miR-197.

The function of miR-197 in oncogenesis and development is very complicated. Fiori et al. reported that miR-197 promotes tumor progression by inhibiting the apoptosis of p53 WT cells in non-small cell lung cancer (NSCLC) ${ }^{10}$ However, it also exerts a tumor suppressor function in multiple myeloma. ${ }^{11}$ Moreover, miR-197 inhibits the proliferation of glioma cells, ${ }^{12}$ but promotes the progression of stomach, ${ }^{13}$ pancreas, ${ }^{14}$ colorectal tumors ${ }^{15}$ and hepatocellular carcinoma. ${ }^{16}$ Clinical studies have shown that miR-197 is of great clinical significance, and it might serve as a promising prognostic biomarker to be used to monitor several types of malignant tumors. For example, miR-197 relates to the poor outcome and distant metastases in patients with NSCLC while its expression is also relevant to the size of the tumor and histopathological classification. ${ }^{17} \mathrm{~A}$ meta-analysis confirmed that compared with a single miRNA, a group of multiple miRNAs may bear greater predictive ability. ${ }^{18}$ Recent studies demonstrated that compared with the normal population, serum miR-197 expression in NSCLC patients is significantly higher, and the expression of miR-145 is remarkably reduced. These changes are related to the clinical stages and pathological classifications of NSCLC patients, and the combination of miR-197 and miR-145 has a high sensitivity for diagnosing NSCLC. ${ }^{19}$ To date, there is no prior research of the function of miR197 in GBC. In this study, the expression of miR-197 in the tumor tissues of GBC patients was found to be significantly higher in GBC tissues, which was consistent with the results of bioinformatics analysis. ${ }^{8}$ Subsequently, in 2 different GBC cell lines, downregulation of miR-197 inhibited the proliferation of GBC cells and promoted apoptosis, while upregulation induced the opposite effect. Therefore, it is possible to elucidate the biological activity of miR-197 in GBC and its regulatory mechanism for the first time.

In the present study, a target of miR-197 was shown to be IGFBP3, which plays an important role in tumor progression through different molecular pathways. On the one hand, IGFBP3 attenuates the signal transduction downstream of IGFR1 induced by IGF1 by interfering with the binding between IGF1 and IGF1R. ${ }^{20}$ The IGF1/IGF1R axis can stimulate epithelial to mesenchymal transition and promote the metastasis of various tumors. ${ }^{21,22}$ On the other hand, IGFBP3 can also inhibit tumor progression in an independent manner. For example, IGFBP3 inhibits head and neck squamous cell carcinoma by blocking the c-jun and c-fos pathways. ${ }^{23}$ It also inhibits the progression of prostate cancer by degrading the NF-kB signaling. ${ }^{24}$ However, the function of IGFBP3 is still controversial. Chan et al. indicated that high expression of IGFBP3 is associated with an increased incidence of colorectal cancer in elderly males, which is independent of IGF1. ${ }^{25}$ It is suggested that IGFBP3 may have differential actions in different tumors. To date, however, no studies have reported whether IGFBP3 is involved in the progression of GBC. Our results indicated that upregulation of miR-197 caused IGFBP3 inhibition, that miR-197 downregulation led to IGFBP3 overexpression, and finally silencing IGFBP3 could partly reverse the effect induced by miR-197 suppression. Hence, it can be concluded that IGFBP3 plays a role in inhibiting $\mathrm{GBC}$ progression. In the current study, miR-197 upregulation caused a decrease in the expression of IGFBP3, which promoted the upregulation of $\mathrm{p}-\mathrm{ERK} 1 / 2$, $\mathrm{p}-\mathrm{AKT}$ and $\mathrm{Bcl}-2$ in GBC cells, and downregulation of Bax and caspase-3, all of which could be reversed after downregulation of miR-197.

Previous studies have found that IGFBP3 plays an important role in regulating tumor cell proliferation and apoptosis. Chen et al. found that IGFBP3 is an indicator of poor prognosis in patients with glioma, and reducing its expression can inhibit tumor growth by inducing apoptosis of glioma cells. ${ }^{26}$ Day et al. found that using IGFBP3 siRNA to treat NSCLC cells with TNFAIP8 knockdown can upregulate pAKT levels and promote cell proliferation. ${ }^{27}$ By blocking the IGF1 signal cascade, it was possible to confirm to a certain degree that overexpression of IGFBP3 can induce apoptosis in NSCLC cells in vitro and enhance cellular response to cisplatin. ${ }^{28}$ In lung adenocarcinoma and hepatocellular carcinoma, testis-specific protein Y-linked 1 (TSPY1) can directly bind to the promoter of $I G F B P 3$ to inhibit its expression. Downregulation of IGFBP3 activates the PI3K/AKT and RAS/RAF/MEK/ ERK signaling pathway, thereby promoting tumor cell proliferation. ${ }^{29}$ Overall, the mechanisms of IGFBP3 on tumor cell regulation varies significantly, and that this difference is likely tumor-specific.

\section{Clinical implications}

The results of the five-year survival analysis of patients recruited to the present study suggest that miR-197 expression is associated with poor prognosis in GBC. This implies that increased expression of miR-197 can be used clinically as a prognostic marker. Since the downregulation of miR197 inhibited proliferation and promoted apoptosis of GBC cells, it is tempting to speculate that the miR-197-IGFBP axis might serve as a therapeutic target in patients with GBC. 


\section{Limitations}

In this study, patients with miR-197 overexpression in tumor tissues had short OS, but OS was not consistent with clinicopathological characteristics. The reason could be attributed to the past surgical history of the patients. Therefore, there was a lack of diversity of clinical stages amongst that recruited patients in the 2 groups of high or low miR197 expression. In addition, the sample size of this study is relatively small, which also diminishes the quality of this study. The best way to determine the impact of miR-197 on GBC cell lines would be to determine the IGFBP3 function in cell proliferation. Therefore, further research could focus on identifying the effect of IGFBP3 on cell proliferation. Moreover, detection of miR-197 levels in patients' peripheral blood can also be performed, which would further support miR-197 as a promising clinical marker.

\section{Conclusions}

Our study demonstrated that the miR-197/IGFBP3 axis regulates the proliferation and apoptosis of GBC cells. Downregulation of miR-197 inhibits the proliferation and promotes the apoptosis of GBC cells, indicating its potential use as a molecular target in the treatment of GBC.

\section{ORCID iDs}

Jinglin Cheng (D) https://orcid.org/0000-0001-7651-4188 Li Tong (D) https://orcid.org/0000-0002-9060-9076 Heping Zuo (D) https://orcid.org/0000-0001-6354-0555 Jingrong Li (i) https://orcid.org/0000-0002-3945-6713

\section{References}

1. Gamboa AC, Maithel SK. The Landmark Series: Gallbladder cancer. Ann Surg Oncol. 2020;27(8):2846-2858. doi:10.1245/s10434-020-08654-9

2. Mao W, Deng F, Wang D, Gao L, Shi X. Treatment of advanced gallbladder cancer: A SEER-based study. Cancer Med. 2020;9(1):141-150. doi:10.1002/cam4.2679

3. Baiu I, Visser B. Gallbladder cancer. JAMA. 2018;320(12):1294. doi:10. 1001/jama.2018.11815
4. Sharma A, Sharma KL, Gupta A, Yadav A, Kumar A. Gallbladder cancer epidemiology, pathogenesis and molecular genetics: Recent update. World J Gastroenterol. 2017;23(22):3978-3998. doi:10.3748/wjg.v23. i22.3978

5. Shroff RT, Kennedy EB, Bachini M, et al. Adjuvant therapy for resected biliary tract cancer: ASCO Clinical Practice Guideline. J Clin Oncol. 2019;37(12):1015-1027. doi:10.1200/JCO.18.02178

6. Chandra V, Kim JJ, Mittal B, Rai R. MicroRNA aberrations: An emerging field for gallbladder cancer management. World $J$ Gastroenterol. 2016;22(5):1787-1799. doi:10.3748/wjg.v22.i5.1787

7. Wang DD, Chen X, Yu DD, et al. miR-197: A novel biomarker for cancers. Gene. 2016;591(2):313-319. doi:10.1016/j.gene.2016.06.035

8. Goeppert B. Truckenmueller F, Ori A, et al. Profiling of gallbladder carcinoma reveals distinct miRNA profiles and activation of STAT1 by the tumor suppressive miRNA-145-5p. Sci Rep. 2019;9(1):4796. doi:10. 1038/s41598-019-40857-3

9. Zhou N, Sun Z, Li N, et al. miR197 promotes the invasion and migration of colorectal cancer by targeting insulin-like growth factorbinding protein 3. Oncol Rep. 2018;40(5):2710-2721. doi:10.3892/or.2018.6640

10. Fiori ME, Barbini C, Haas TM, et al. Antitumor effect of miR-197 targeting in p53 wild-type lung cancer. Cell Death Differ. 2014;21(5):774-782. doi:10.1038/cdd.2014.6

11. Yang Y, Li F, Saha MN, Abdi J, Qiu L, Chang H. miR-137 and miR-197 induce apoptosis and suppress tumorigenicity by targeting MCL-1 in multiple myeloma. Clin Cancer Res. 2015;21(10):2399-2411. doi:10. 1158/1078-0432.CCR-14-1437

12. Tian LQ, Liu EQ, Zhu XD, Wang XG, Li J, Xu GM. MicroRNA-197 inhibits cell proliferation by targeting GAB2 in glioblastoma. Mol Med Rep. 2016;13(5):4279-4288. doi:10.3892/mmr.2016.5076

13. Xu L, Hou Y, Tu G, et al. Nuclear Drosha enhances cell invasion via an EGFR-ERK1/2-MMP7 signaling pathway induced by dysregulated miRNA-622/197 and their targets LAMC2 and CD82 in gastric cancer. Cell Death Dis. 2017;8(3):e2642. doi:10.1038/cddis.2017.5

14. Hamada S, Satoh K, Miura S, et al. miR-197 induces epithelial-mesenchymal transition in pancreatic cancer cells by targeting p120 catenin. J Cell Physiol. 2013;228(6):1255-1263. doi:10.1002/jcp.24280

15. Huang $Q, M a B, S u Y$, et al. miR-197-3p represses the proliferation of prostate cancer by regulating the VDAC1/AKT/beta-catenin signaling axis. Int J Biol Sci. 2020;16(8):14171426. doi:10.7150/ijbs.42019

16. Dai W, Wang C, Wang F, et al. Anti-miR-197 inhibits migration in HCC cells by targeting KAl 1/CD82. Biochem Biophys Res Commun. 2014; 446(2):541-548. doi:10.1016/j.bbrc.2014.03.006

17. Mavridis K, Gueugnon F, Petit-Courty A, et al. The oncomiR miR-197 is a novel prognostic indicator for non-small cell lung cancer patients. Br J Cancer. 2015;112(9):1527-1535. doi:10.1038/bjc.2015.119

18. Wang $\mathrm{R}$, Wen $\mathrm{H}$, Xu $\mathrm{Y}$, et al. Circulating microRNAs as a novel class of diagnostic biomarkers in gastrointestinal tumors detection: A meta-analysis based on 42 articles. PLoS One. 2014;9(11):e113401. doi:10.1371/journal.pone.0113401

19. Sui A, Zhang X, Zhu Q. Diagnostic value of serum miR197 and miR145 in non-small cell lung cancer. Oncol Lett. 2019;17(3):3247-3252. doi:10. 3892/ol.2019.9958
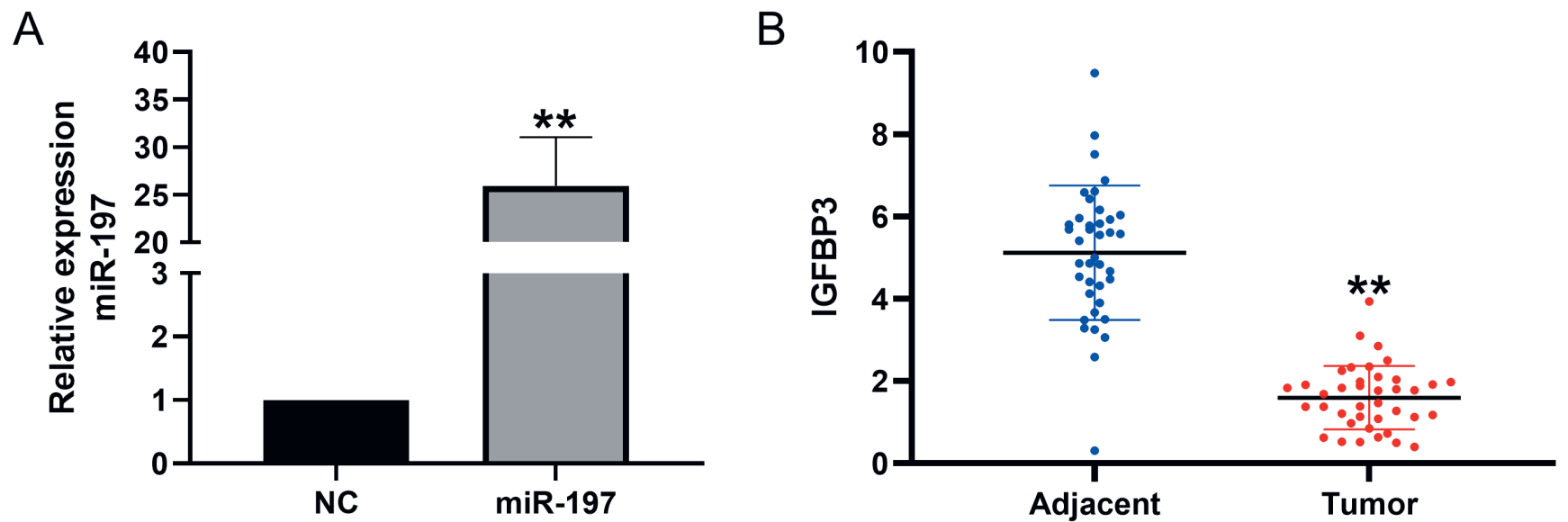

Supplementary Fig. 6. A. After miR-197 transfection, the expression of miR-197 in MZ-CHA-1 cells increased significantly $\left({ }^{* *}\right.$ compared with NC, p < 0.01); B. The expression of IGFBP3 in GBC tissues and adjacent normal tissues was detected using qRT-PCR (** Compared with the adjacent group, p <0.01) 
20. Valadez-Bustos N, Escamilla-Silva EM, García-Vázquez FJ, GallegosCorona MA, Amaya-Llano SL, Ramos-Gómez M. Oral administration of microencapsulated B. Longum BAA-999 and lycopene modulates IGF-1/IGF-1R/IGFBP3 protein expressions in a colorectal murine model. Int J Mol Sci. 2019;20(17):4725. doi:10.3390/ijms20174275

21. Wang XH, Wu HY, Gao J, Wang XH, Gao TH, Zhang SF. IGF1R facilitates epithelial-mesenchymal transition and cancer stem cell properties in neuroblastoma via the STAT3/AKT axis. Cancer Manag Res. 2019;11:5459-5472. doi:10.2147/CMAR.S196862

22. Neuzillet $Y$, Chapeaublanc E, Krucker C, et al. IGF1R activation and the in vitro antiproliferative efficacy of IGF1R inhibitor are inversely correlated with IGFBP5 expression in bladder cancer. BMC Cancer. 2017;17(1):636. doi:10.1186/s12885-017-3618-5

23. Lee HJ, Lee JS, Hwang SJ, Lee HY. Insulin-like growth factor binding protein-3 inhibits cell adhesion via suppression of integrin beta4 expression. Oncotarget. 2015;6(17):15150-15163. doi:10.18632/oncotarget.3825

24. Kim H, Datta A, Talwar S, Saleem SN, Mondal D, Abdel-Mageed AB. Estradiol-ERbeta2 signaling axis confers growth and migration of CRPC cells through TMPRSS2-ETV5 gene fusion. Oncotarget. 2017;8(38): 62820-62833. doi:10.18632/oncotarget.11355
25. Chan YX, Alsonso H, Chubb SAP, et al. Higher IGFBP3 is associated with increased incidence of colorectal cancer in older men independently of IGF1. Clin Endocrinol (Oxf). 2018;88(2):333-340. doi:10.1111/ cen.13499

26. Chen $\mathrm{CH}$, Chen $\mathrm{PY}$, Lin $\mathrm{YY}$, et al. Suppression of tumor growth via IGFBP3 depletion as a potential treatment in glioma. J Neurosurg. 2019;132(1):168-179. doi:10.3171/2018.8.JNS181217

27. Day TF, Kallekury BVS, Ross JS, et al. Dual targeting of EGFR and IGF1R in the TNFAIP8 knockdown non-small cell lung cancer cells. Mol Cancer Res. 2019;17(5):1207-1219. doi:10.1158/1541-7786.MCR-18-0731

28. Wang YA, Sun Y, Palmer J, et al. IGFBP3 modulates lung tumorigenesis and cell growth through IGF1 signaling. Mol Cancer Res. 2017; 15(7):896-904. doi:10.1158/1541-7786.MCR-16-0390

29. Tu W, Yang B, Leng $X$, et al. Testis-specific protein, Y-linked 1 activates $\mathrm{PI} 3 \mathrm{~K} / \mathrm{AKT}$ and RAS signaling pathways through suppressing IGFBP3 expression during tumor progression. Cancer Sci. 2019;110(5): 1573-1586. doi:10.1111/cas.13984 